\title{
BMJ Open Prevalence and associated factors of active smoking among individuals living with hypertension and/or diabetes in Africa: a systematic review and meta-analysis protocol
}

\author{
Guy S Wafeu, ${ }^{1}$ Aurel T Tankeu, ${ }^{1}$ Francky Teddy A Endomba, ${ }^{1}$ \\ Jobert Richie Nansseu, ${ }^{2,3}$ Arnaud D Kaze, ${ }^{4}$ Jean Joel Bigna,, \\ Jean Jacques Noubiap ${ }^{7}$
}

To cite: Wafeu GS, Tankeu AT, Endomba FTA, et al. Prevalence and associated factors of active smoking among individuals living with hypertension and/or diabetes in Africa: a systematic review and metaanalysis protocol. BMJ Open 2017;7:e015444. doi:10.1136/ bmjopen-2016-015444

- Prepublication history for this paper is available online. To view these files, please visit the journal online (http://dx.doi. org/10.1136/bmjopen-2016015444).

Received 5 December 2016 Accepted 28 July 2017

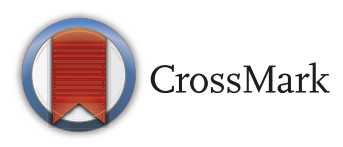

For numbered affiliations see end of article.

Correspondence to Dr Jean Jacques Noubiap; noubiapjj@yahoo.fr

\section{ABSTRACT}

Introduction Tobacco use significantly increases cardiovascular complications in people living with hypertension and/or diabetes. We aim to summarise data on the prevalence and factors associated with active smoking in these conditions in Africa.

Method and analysis We will search PubMed, Embase, Google Scholar and African Journals Online for relevant abstracts of studies on active smoking in individuals living with diabetes and/or hypertension published from 1 January 2000 to 31 December 2016, with no language restriction. Additionally, relevant unpublished papers and conference proceedings will be checked, as well as references of included articles. Two investigators will independently screen, select studies, extract data and assess the risk of bias in each study. Data will be analysed using Stata software (Stata V.14, Texas, USA). The study-specific estimates will be pooled through a random-effects meta-analysis model to obtain an overall summary estimate of the prevalence of smoking across studies. Also, we will assess factors associated to smoking. Heterogeneity of studies will be evaluated by the $\chi 2$ test on Cochrane's $Q$ statistic. Funnel plots analysis and Egger's test will be done to detect publication bias. Results will be presented by geographic region (central, eastern, northern, southern and western Africa). A p value less than 0.05 will be considered significant for factors associated to smoking.

Ethics and dissemination This study is based on published data, and therefore ethical approval is not a requirement. This systematic review and meta-analysis is expected to serve as a basis for designing cost-effective interventions to reduce and prevent smoking in patients with diabetes and/ or hypertension, and as a guide for future research based on the remaining gaps. The final report of this study in the form of a scientific paper will be published in peer-reviewed journals. Findings will further be presented at conferences and submitted to relevant health authorities.

\section{INTRODUCTION}

\section{Rationale}

In 2015, cardiovascular diseases (CVDs) were responsible for approximately 18 million
Strengths and limitations of this study

- This will be the first systematic review and metaanalysis summarising the prevalence of active smoking among individuals living with hypertension and/or diabetes in Africa.

- Strong and robust statistical methods will be used for summarising data.

- This review would be limited by the predominance of clinic-based studies and the poor quality data.

- For determining risk factors associated with active smoking, cross-sectional studies are not best design. The review would be therefore limited for this specific purpose since most of studies could have cross-sectional design.

deaths worldwide, representing the leading cause of death. ${ }^{1}$ That figure is increasing in both low/middle-income countries and developed countries as risk factors for the disease continue to increase in both contexts. ${ }^{1}$ In sub-Saharan Africa (SSA) especially, CVDs caused nearly 1 million deaths in 2013, representing $38.3 \%$ of non-communicable disease-related deaths and $11.3 \%$ of all-cause mortality. ${ }^{2}$ Intriguingly, these deaths due to CVDs in SSA occur at a younger age as compared with the rest of the world. ${ }^{3}$

Hypertension, diabetes mellitus, hypercholesterolaemia and smoking are the four major modifiable traditional cardiovascular risk factors. ${ }^{4}$ In fact, $80 \%$ to $95 \%$ of patients who experienced a fatal or non-fatal cardiovascular event had at least one of these four major cardiovascular risk factors. ${ }^{56}$ In 2010, the two leading risk factors for global disease burden were high blood pressure (7\% of global disability-adjusted life years) and tobacco smoking $(6.3 \%$ of global 
disability-adjusted life years). ${ }^{7}$ In patients with hypertension or diabetes, smoking appears to be a significant and independent risk factor for all-cause, CVD and non-CVD morbidity and mortality. ${ }^{8}$ It remains the cause of 6 million preventable deaths per year globally. ${ }^{9}$

World Health Assembly endorsed a voluntary global target of a $30 \%$ relative reduction in tobacco use worldwide among people aged 15 years or older by 2025 (with 2010 levels as baseline). ${ }^{9}$ From 2000 to 2010 , prevalence of tobacco smoking fell in more than $70 \%$ of countries, mostly in those with high incomes. In 2012, the global prevalence of current tobacco smoking among adults was 22\%.$^{9}$ Furthermore, a rapid increase is projected by 2025 in African and eastern Mediterranean low-income and middle-income countries. ${ }^{10}$ Tobacco use increases the risk of CVD and premature death, and smoking cessation is an important part of hypertension and diabetes management. ${ }^{91112}$ However, the burden and magnitude of active tobacco smoking is not well known in Africans suffering from hypertension and diabetes, which could inform on how much efforts should be made to reduce/lessen these growing threats. We present here the protocol for a systematic review and meta-analysis to estimate the prevalence of active smoking among African patients with hypertension and/or diabetes, as well as associated factors. Results are intended to help in emphasising on the need to control and prevent smoking among those patients, and to serve as basis for designing effective interventions accordingly. The results may also provide a robust basis for monitoring future trends.

\section{Objective}

To conduct a systematic review and meta-analysis in order to estimate the prevalence of active smoking among individuals living with hypertension and/or diabetes in Africa, as well as its associated factors.

\section{METHODS}

\section{Eligibility criteria}

Inclusion criteria

1. Population: persons living with hypertension and/ or diabetes aged more than 15 years residing in Africa continent. Hypertension will be considered in the presence of systolic blood pressure $\geq 140 \mathrm{~mm}$ $\mathrm{Hg}$ and/or diastolic blood pressure $\geq 90 \mathrm{~mm} \mathrm{Hg}$ or any antihypertensive treatment. ${ }^{13}$ Diabetes will be considered using the following diagnostic criteria: A1c haemoglobin $\geq 6.5 \%$ or fasting plasma glucose $\geq 126 \mathrm{mg} / \mathrm{dL}(7.0 \mathrm{mmol} / \mathrm{L})$ or 2 hours plasma glucose $\geq 200 \mathrm{mg} / \mathrm{dL} \quad(11.1 \mathrm{mmol} / \mathrm{L})$ or random plasma glucose $\geq 200 \mathrm{mg} / \mathrm{dL}(11.1 \mathrm{mmol} / \mathrm{L})$ in the presence of classic symptoms of hyperglycaemia. ${ }^{14}$ All types of diabetes will be considered.

2. Type of studies: cross-sectional, case-control or cohort studies.

3. Outcome: active smoking which will be defined as current use of any tobacco product in either smoked or smokeless form. ${ }^{9}$
4. Type of data: prevalence of active smoking or enough data to compute this estimate and factors associated with active smoking.

5. Studies published in any language and unpublished studies (trial registries, conference proceedings, dissertations, monographs and reports held by government agencies, academics).

\section{Exclusion criteria}

1. Studies on non-systemic hypertension (intracranial hypertension, pulmonary hypertension) or studies on gestational diabetes.

2. Studies conducted among populations of African origin residing outside of Africa.

3. Studies including adult and paediatric populations in which it will not be possible to extract data for adults after contacting the corresponding authors.

4. Case series with small sample size (less than 50 participants), letters, reviews, commentaries and editorials.

5. Studies lacking key data and/or explicit method description.

6. Duplicates: for studies published in more than one paper, the most comprehensive one reporting the largest sample size will be considered.

7. Studies with serious ethical issues.

8. Studies whose full data will not be accessible even after request from the authors.

\section{Information sources and search strategy}

This systematic review and meta-analysis will follow the Institutes of Medicine Standards for Systematic Reviews. ${ }^{15}$ Reporting will align to the guidelines set out by Preferred Reporting Items for Systematic Reviews and Meta-Analyses (PRISMA) group. ${ }^{16}$ The search strategy will be implemented in two stages:

1. Relevant abstracts published on the prevalence and associated factors of active smoking in African individuals living with hypertension and/or diabetes will be identified after searching PubMed/Medline, Excerpta Medica Database (EMBASE) and African Journals Online. The search will include studies from 1 January 2000 to 31 December 2016. Both text words and medical subject heading terms will be used. Key search terms will be 'Africa', 'hypertension', 'diabetes' and 'smoking'. We will also use individual country names for the 54 African countries as additional key search terms in order to obtain more abstracts on the subject. The main search strategy is shown in table 1 . An expert liberian will conduct searches in different databases.

2. The titles and abstracts of all eligible papers will be reviewed and full articles will be accessed through PubMed, Google Scholar, HINARI or journals' websites. The references of all relevant research articles and review papers will also be scrutinised for additional potential data sources, and their full texts will be accessed in a similar way. The authors whose full text papers will not be accessible by the numerous 


\begin{tabular}{|c|c|}
\hline Search & Search terms \\
\hline 1 & $\begin{array}{l}\text { Smoking [tw] OR tobacco [tw] OR snuff [tw] OR cigarette [tw] OR Cigar [tw] OR pipe [tw] OR chewing [tw] OR } \\
\text { Smoking [MeSH terms] OR nicotine [tw] OR tabacum [tw] OR nicotiana [tw] OR waterpipe [tw] OR e-cig [tw] OR } \\
\text { e-cigarette [tw] }\end{array}$ \\
\hline 2 & $\begin{array}{l}\text { (Hypertension [tw] OR Hypertension [MeSH terms] OR high blood pressure [tw] OR systolic hypertension [tw] OR } \\
\text { diastolic hypertension [tw] OR Hypertensive patients OR Raised blood pressure ([tw]) }\end{array}$ \\
\hline 4 & (\# 1 AND \# 3) OR (\# 2 AND \# 3) \\
\hline 5 & 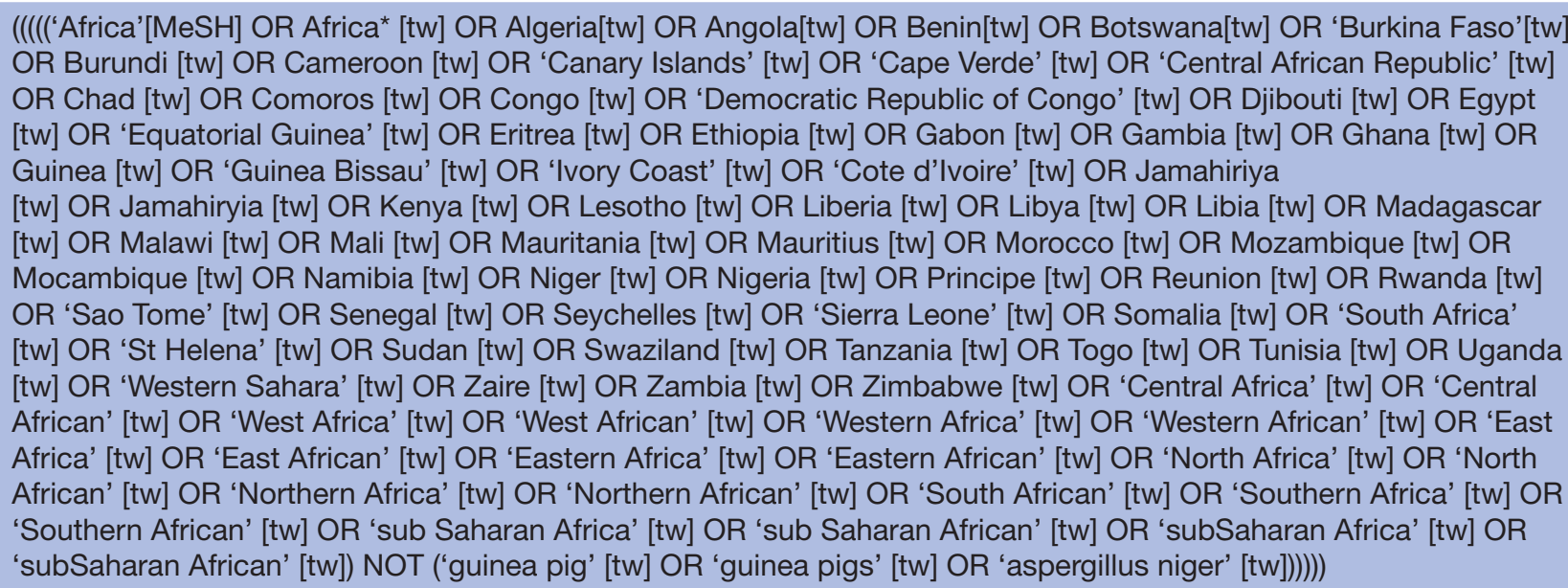 \\
\hline 6 & \# 4 AND \# 5 \\
\hline
\end{tabular}

internet-based sources will be directly contacted to provide them. In case of no feedback from these authors, the corresponding studies will be excluded.

\section{Selection process}

Assessment of eligible papers will be independently conducted by two review authors, using an assessment guide to ensure that the selection criteria are reliably applied by each of these authors. The same authors will independently assess the full texts of records deemed relevant or potentially relevant for eligibility; any disagreement between them will be resolved by a third author. Records resulting from search strategy will be transferred to EndNote X7 for selection of studies based on title and abstract and removing of duplicates. Eligible studies in other languages will be translated using Google Translate and considered for inclusion. Agreement between review authors will be measured using the Cohen's Kappa statistic. $^{17}$

\section{Data collection process and data items}

A data extraction sheet will be used to collect information about the country, the African subregion, year of publication, type of publication, study design, study population, number of participants, mean/median age of participants, male proportion, prevalence of active smoking and predictive factors whenever available, and the frequency of different forms of tobacco (cigarettes, chew, bidis, chew, cigars, E-cigarette, hookah, pipe, snuff, water pipe).
Where prevalence of smoking or information for calculating it (eg, sample size, number of smokers) is lacking, we will directly contact the corresponding author to request the information. In case of multinational studies, we will separate the results to show the prevalence and associated factors within individual countries. Where it will not be possible to disaggregate the data by country, the study will be presented as one and the countries in which the study was done will be shown.

\section{Assessment of methodological quality and data reporting}

The Newcastle-Ottawa Scale (NOS) for assessing the quality of non-randomised studies in meta-analyses will be used to assess the quality of case-control and cohort studies. ${ }^{18}$ A modified version of the NOS will be used for cross-sectional studies. Risk of bias and quality scores will be presented in a table.

\section{Data synthesis and analysis}

Data will be analysed using Stata software (StataV.14). A stratified meta-analysis will be conducted for data obtained from studies depending on the definition of active smoking in those studies. Standard errors for the study-specific estimates will first be determined from the point estimate and the appropriate denominators, assuming a binominal distribution. Then the study-specific estimates will be pooled through a random-effects meta-analysis model to obtain an overall summary estimate of the across studies, after stabilising the variance of individual studies using the 
Freeman-Tukey double arcsine transformation. ${ }^{19}$ Score method will be used to compute the study-specific CI. ${ }^{20} \mathrm{~A}$ metaregression will be performed to assess factors associated to smoking. If it is not possible to summarise data, a narrative review will be conducted for associated factors. A $p$ value less than 0.05 will be considered significant for factors associated to smoking.

Heterogeneity will be evaluated by the $\chi 2$ test on Cochrane's $Q$ statistic, ${ }^{21}$ which is quantified by $\mathrm{I}^{2}$ values, ${ }^{22}$ assuming that $\mathrm{I}^{2}$ values of $25 \%, 50 \%$ and $75 \%$, represent low, medium and high heterogeneity, respectively. Where substantial heterogeneity will be detected, a subgroup analysis will be performed to detect its possible sources using the following grouping variables: age group, sex, geographical area (central, eastern, northern, southern and western Africa), study quality, baseline disease (diabetes vs hypertension) and definition of smoking. Subgroups comparisons then used the Q-test based on the analysis of the variance. Inter-rater agreement for study inclusion will be assessed using the Cohen's $\kappa$ coefficient. ${ }^{23}$ Funnel plots analysis and Egger's test will be done to detect publication bias. A p-Egger test $<0.1$ will be consider indicative of statistically significant publication bias. ${ }^{24}$ Results will be presented by geographical region (central, eastern, northern, southern and western Africa).

\section{Results reporting and presentation}

The study selection process will be summarised using a flow diagram. Reasons for studies' exclusion will be described. This review will follow the guidelines set out by the PRISMA group. ${ }^{16}$ Quantitative data will be presented in evidence tables of individual studies as well as in summary tables and funnel plots where appropriate. We will examine prevalence of active smoking and associated factors by region, time period and disease-specific populations depending on the data available. We plan to report on quality scores and risk of bias for each eligible study. This may be tabulated and accompanied by narrative summaries.

\section{Ethics and dissemination}

This study is based on published data, and therefore ethical approval is not a requirement. This systematic review and meta-analysis is expected to serve as a basis for designing cost-effective interventions to reduce and prevent smoking in patients with hypertension and/or diabetes, and as a guide for future research based on the remaining gaps. The final report of this study in the form of a scientific paper will be published in peer-reviewed journals. Additionally, findings will be presented at conferences and submitted to relevant health authorities. We also plan to update the review in the future to monitor changes and guide health service and policy solutions.

\section{CONCLUSIONS}

Smoking is a major risk factor for CVDs which are actually rising in Africa. In patients with hypertension and/ or diabetes, active smoking independently and significantly increase the risk for complications. It is therefore necessary to introduce cost-effective interventions in order to reduce smoking in those populations, remembering that it has been projected an increase in tobacco smoking burden in Africa. Prior to these strategies, accurate epidemiological data should be obtained. We anticipate that this review would guide policy, practice and research by providing information on the magnitude of smoking among patients with hypertension and/or diabetes and associated factors.

There are some possible limitations to this review, among which the poor quality of data when available, the heterogeneity of studies which will make further analysis difficult and the predominance of cross-sectional studies, making it difficult to determine smoking-associated factors. Other drawbacks could include the non-random selection of participants and the under-representation of African subregions. These problems have already been highlighted by previous studies on non-communicable diseases in Africa. ${ }^{25} 26$

\section{Author affiliations}

${ }^{1}$ Department of internal medicine and specialties, Faculty of Medicine and Biomedical Sciences, Yaoundé, Cameroon

${ }^{2}$ Department of Public Health, Faculty of Medicine and Biomedical

Sciences,University of Yaoundé 1, Yaoundé, Cameroon

${ }^{3}$ Sickle cell disease unit, Mother and Child Centre of the Chantal Biya Foundation, Yaoundé, Cameroon

${ }^{4}$ Brigham and women's hospital, Harvard Medical School, Boston, MA, USA ${ }^{5}$ Department of Epidemiology and Public Health, Centre Pasteur of Cameroon, Yaoundé, Cameroon

${ }^{6}$ Faculty of Medicine, University of Paris Sud XI, Le Kremlin Bicêtre, France ${ }^{7}$ Department of Medicine, Groote Schuur Hospital and University of Cape Town, Cape Town, South Africa

Contributors JJN, GSW, ATT and FTEA conceived and designed the protocol. GSW drafted the manuscript. ATT, JJB, JRN, FTEA, ADK and JJN critically revised the manuscript for methodological and intellectual content. JJN is the guarantor of the review. All authors approved the final version of this manuscript.

Competing interests None declared.

Patient consent Detail has been removed from this case description/these case descriptions to ensure anonymity. The editors and reviewers have seen the detailed information available and are satisfied that the information backs up the case the authors are making.

Provenance and peer review Not commissioned; externally peer reviewed.

Open Access This is an Open Access article distributed in accordance with the Creative Commons Attribution Non Commercial (CC BY-NC 4.0) license, which permits others to distribute, remix, adapt, build upon this work non-commercially, and license their derivative works on different terms, provided the original work is properly cited and the use is non-commercial. See: http://creativecommons.org/ licenses/by-nc/4.0/

(C) Article author(s) (or their employer(s) unless otherwise stated in the text of the article) 2017. All rights reserved. No commercial use is permitted unless otherwise expressly granted.

\section{REFERENCES}

1. GBD 2015 Mortality and Causes of Death Collaborators. Global, regional, and national life expectancy, all-cause mortality, and causespecific mortality for 249 causes of death, 1980-2015: a systematic analysis for the Global Burden of Disease Study 2015. Lancet 2016;388:1459-544.

2. Mensah GA, Roth GA, Sampson UK, et al. Mortality from cardiovascular diseases in sub-Saharan Africa, 1990-2013: a systematic analysis of data from the Global Burden of Disease Study 2013. Cardiovasc J Afr 2015;26(2 Suppl 1):S6-S10. 
3. Moran A, Forouzanfar M, Sampson U, et al. The epidemiology of cardiovascular diseases in sub-Saharan Africa: the Global Burden of Diseases, Injuries and Risk Factors 2010 Study. Prog Cardiovasc Dis 2013;56:234-9.

4. Canto JG, Iskandrian AE. Major risk factors for cardiovascular disease: debunking the "Only 50\%" myth. JAMA 2003;290:947.

5. Khot UN, Khot MB, Baizer CT, et al. Prevalence of conventional risk factors in patients with coronary heart disease. JAMA 2003;290:898.

6. Greenland P, Knoll MD, Stamler J, et al. Major risk factors as antecedents of fatal and nonfatal coronary heart disease events. JAMA 2003;290:891.

7. Lim SS, Vos T, Flaxman AD, et al. A comparative risk assessment of burden of disease and injury attributable to 67 risk factors and risk factor clusters in 21 regions, 1990-2010: a systematic analysis for the Global Burden of Disease Study 2010. Lancet 2012;380:2224-60.

8. Fagard RH. Smoking amplifies cardiovascular risk in patients with hypertension and diabetes. Diabetes Care 2009;32:S429-S431.

9. World Health Organization. Global status report on noncommunicable diseases 2014: attaining the nine global noncommunicable diseases targets; a shared responsibility. Geneva: World Health Organization 2014.

10. Bilano V, Gilmour S, Moffiet T, et al. Global trends and projections for tobacco use, 1990-2025: an analysis of smoking indicators from the WHO Comprehensive Information Systems for Tobacco Control. Lancet 2015;385:966-76.

11. Mancia G, De Backer G, Dominiczak A, et al. Management of Arterial Hypertension of the European Society of HypertensionEuropean Society of Cardiology. Guidelines for the Management of Arterial Hypertension: The Task Force for the Management of Arterial Hypertension of the European Society of Hypertension (ESH) and of the European Society of Cardiology (ESC). J Hypertens 2007;25:1105-87.

12. Rydén L, Grant PJ, Anker SD, et al. ESC Guidelines on diabetes, prediabetes, and cardiovascular diseases developed in collaboration with the EASD: the Task Force on diabetes, pre-diabetes, and cardiovascular diseases of the European Society of Cardiology (ESC) and developed in collaboration with the European Association for the Study of Diabetes (EASD). Eur Heart J 2013;34:3035-87.
13. James PA, Oparil S, Carter BL, et al. 2014 evidence-based guideline for the management of high blood pressure in adults: report from the panel members appointed to the Eighth Joint National Committee (JNC 8). JAMA 2014;311:507.

14. American Diabetes Association. Diagnosis and classification of diabetes mellitus. Diabetes Care 2012;35:S64-71.

15. Institute of Medicine (U.S.). Eden J, ed. Finding what works in health care: standards for systematic reviews. Washington, D.C: National Academies Press, 2011:317.

16. Moher D, Liberati A, Tetzlaff J, et al. Preferred reporting items for systematic reviews and meta-analyses: the PRISMA statement. BMJ 2009;339:b2535.

17. McHugh ML. Interrater reliability: the kappa statistic. Biochem Med 2012;22:276-82.

18. Wells G, Shea B, O'Connell D, et al. The Newcastle-Ottawa Scale (NOS) for assessing the quality of nonrandomised studies in metaanalyses [Internet]. Ottawa Hospital Research Institute 2014 http:// www.ohri.ca/programs/clinical_epidemiology/oxford.asp (cited 2016 Oct 25).

19. Barendregt JJ, Doi SA, Lee YY, et al. Meta-analysis of prevalence. $J$ Epidemiol Community Health 2013;67:974-8.

20. Wilson EB, Inference P. the Law of Succession, and Statistical Inference. J Am Stat Assoc 1927;22:209.

21. Cochran WG. The Combination of Estimates from Different Experiments. Biometrics 1954;10:101.

22. Higgins JP, Thompson SG. Quantifying heterogeneity in a metaanalysis. Stat Med 2002;21:1539-58.

23. Landis JR, Koch GG. The measurement of observer agreement for categorical data. Biometrics 1977;33:159.

24. Egger M, Davey Smith G, Schneider M, et al. Bias in meta-analysis detected by a simple, graphical test. BMJ 1997;315:629-34.

25. Noubiap JJ, Naidoo J, Kengne AP. Diabetic nephropathy in Africa: A systematic review. World J Diabetes 2015;6:759.

26. Kayima J, Wanyenze RK, Katamba A, et al. Hypertension awareness, treatment and control in Africa: a systematic review. BMC Cardiovasc Disord 2013;13:54. 